\title{
ON THE RESTRICTED MEAN VALUE PROPERTY
}

\author{
P. C. FENTON
}

\begin{abstract}
Suppose that $u$ is continuous in the open unit disc and has the restricted mean value property. It is shown that if $u$ has finite boundary limits almost everywhere, and if $u$ possesses a harmonic majorant and minorant, the difference between which has finite radial upper limits everywhere, then $u$ is harmonic.
\end{abstract}

1. Introduction. A function $u(z)$ in the open unit disc $\Delta(0,1)$ has the restricted mean-value ( $\mathrm{rmv}$ ) property if for each $z \in \Delta(0,1)$ there is a positive number $\rho=\rho(z)<1-|z|$ such that

$$
\frac{1}{2 \pi} \int_{-\pi}^{\pi} u\left(z+\rho e^{i \theta}\right) d \theta=u(z)
$$

Here we shall prove

THEOREM 1. Suppose that $u(z)$ is continuous in $\Delta(0,1)$ and has the rmv property. If

(i) $\lim _{z \rightarrow e^{i \theta}} u(z)$ exists for almost all $\theta$, and

(ii) $u(z)$ has a harmonic majorant $h_{2}(z)$ and a harmonic minorant $h_{1}(z)$ such that for all $\theta \in[-\pi, \pi]$

$$
\varlimsup_{r \rightarrow 1}\left\{h_{2}\left(r e^{i \theta}\right)-h_{1}\left(r e^{i \theta}\right)\right\}<\infty
$$

then $u$ is harmonic.

This is an extension of an earlier result of the author [1] in which instead of condition (ii) it is supposed the $u(z)$ is bounded. Both are motivated by problem 11 in J. E. Littlewood's book [2]. It is a genuine extension since $h_{2}(z)-h_{1}(z)$ need not be bounded, as the following theorem shows.

THEOREM 2. Suppose that $h(z)$ is a nonnegative harmonic function in $\Delta(0,1)$ such that

$$
\varlimsup_{r \rightarrow 1} h\left(r e^{i \theta}\right)<\infty
$$

for every $\theta \in[-\pi, \pi]$. Then

$$
h(z)=\frac{1}{2 \pi} \int_{-\pi}^{\pi} H(t) r e\left\{\left(e^{i t}+z\right) /\left(e^{i t}-z\right)\right\} d t,
$$

where $H(t)$ is nonnegative and such that

$$
\varlimsup_{T \downarrow 0} \frac{1}{2 T} \int_{-T}^{T} H(t+\theta) d t<\infty
$$

Received by the editors May 1,1986 .

1980 Mathematics Subject Classification (1985 Revision). Primary 31A05. 
for every $\theta \in[-\pi, \pi]$. Conversely, if $h(z)$ is defined by (1.3), where $H(t)$ is nonnegative and satisfies (1.4) for every $\theta \in[-\pi, \pi]$, then (1.2) holds for every $\theta \in[-\pi, \pi]$.

As will be shown later, Theorem 2 has no natural counterpart for $h^{1}$ functions at least in the sense that there is an $h^{1}$ function $h(z)$ of the form (1.3) satisfying $\varlimsup_{r \rightarrow 1}\left|h\left(r e^{i \theta}\right)\right|<\infty$ for every $\theta \in[-\pi, \pi]$ for which

$$
\varlimsup_{T \downarrow 0}\left|\frac{1}{2 T} \int_{-T}^{T} H(t) d t\right|=+\infty .
$$

2. Two auxiliary functions. Given $\zeta \in \Delta(0,1)$ let $v_{\zeta}(z)$ be the harmonic function in $\Delta(\varsigma, \rho(\varsigma))$ which is the Poisson integral of the boundary values of $u$. Define

$$
V(z)=\sup v_{\varsigma}(z), \quad W(z)=\inf v_{\varsigma}(z)
$$

the supremum and infimum extended over those $\varsigma$ such that $|z-\varsigma|<\rho(\varsigma)$. From condition (ii) both $V(z)$ and $W(z)$ are finite for all $z$ and

$$
h_{2}(z) \geq V(z) \geq v_{z}(z)=u(z) \geq W(z) \geq h_{1}(z) .
$$

These $V$ and $W$ are slightly simpler versions of functions introduced in [1].

The significant properties of $V$ and $W$ are

$$
V(z) \text { is subharmonic and } W(z) \text { is superharmonic, }
$$

and

$$
\text { for a.a. } \theta \in[-\pi, \pi], \lim _{z \rightarrow e^{i \theta}} V(z)=\lim _{z \rightarrow e^{i \theta}} W(z)=\lim _{z \rightarrow e^{i \theta}} u(z) .
$$

To prove these we need

LEMMA. Let $\xi \in \Delta(0,1)$ and let $z_{n} \rightarrow \xi$. If $\varsigma_{n}$ is such that $\left|z_{n}-\varsigma_{n}\right|<\rho\left(\varsigma_{n}\right)$ for all $n$ and if $\rho\left(\zeta_{n}\right)-\left|z_{n}-\zeta_{n}\right| \rightarrow 0$ as $n \rightarrow \infty$, then

$$
v_{\varsigma_{n}}\left(z_{n}\right) \rightarrow u(\xi) \text {. }
$$

Suppose that (2.4) is false for some sequence $\zeta_{n}$ (which may be assumed without loss to be convergent) with limit $\varsigma_{0}$ say. Evidently $\rho_{0} \neq 0$, where $\rho_{0}=\lim \rho\left(\zeta_{n}\right)$, so that $\xi$ is a boundary point of $\Delta\left(\varsigma_{0}, \rho_{0}\right)$.

Given $\varepsilon>0$ let $\delta$ be such that $|u(z)-u(\xi)|<\varepsilon$ for $z$ in $\Delta(\xi, \delta)$. Let $\Gamma$ be a chord of $\Delta(\xi, \delta)$ which is parallel to the tangent to $\Delta\left(\varsigma_{0}, \rho_{0}\right)$ at $\xi$ and which lies outside $\Delta\left(\varsigma_{0}, \rho_{0}\right)$. Let $D$ be the larger part of $\Delta(\xi, \delta)$ cut off by $\Gamma$. Given an upper bound $M$ for $h_{2}(z)$ on $D$, let

$$
H(z)=(M+\varepsilon)\left\{\frac{\pi-\theta}{\pi-\theta_{0}}\right\}+(u(\xi)+\varepsilon)\left\{1-\frac{\pi-\theta}{\pi-\theta_{0}}\right\}, \quad z \in D,
$$

where $\theta=\theta(z)$ is the angle subtended by $\Gamma$ at $z$ and $\theta_{0}$ is the constant value of $\theta$ on the circular part of $\partial D . H(z)$ is harmonic in $D$ with boundary values $u(\xi)+\varepsilon$ on $\Gamma$ and $M+\varepsilon$ on $\partial D \backslash \Gamma$. It follows that for all large $n, v_{\varsigma_{n}}(z) \leq H(z)$ in $D_{n}=D \cap \Delta\left(\zeta_{n}, \rho\left(\zeta_{n}\right)\right)$. For when $n$ is large, $\partial D_{n}$ consists of a part of $\partial D \backslash \Gamma$, on which $H(z)=M+\varepsilon \geq v_{\varsigma_{n}}(z)$, together with the part of the boundary of $\Delta\left(\varsigma_{n}, \rho\left(\varsigma_{n}\right)\right)$ that lies in $D$, and there $v_{\varsigma_{n}}(z)=u(z)<u(\xi)+\varepsilon \leq H(z)$. Hence 
$\varlimsup v_{\zeta_{n}}\left(z_{n}\right) \leq H(\xi)$ and thus, allowing $D$ to contract to a half-disc, $\varlimsup$ lim $v_{\zeta_{n}}\left(z_{n}\right) \leq$ $u(\xi)+\varepsilon$. A similar argument gives $\underline{\lim } v_{\zeta_{n}}\left(z_{n}\right) \geq u(\xi)-\varepsilon$. Since $\varepsilon$ is arbitrary (2.4) follows, contradicting the assumption and proving the lemma.

PROOF OF (2.2). $V(z)$ is upper-semicontinuous. For otherwise there is a point $\xi$ and a sequence $z_{n} \rightarrow \xi$ such that $V\left(z_{n}\right) \rightarrow V_{0}>V(\xi)$. It follows that there is another sequence $\zeta_{n}$ such that $v_{\zeta_{n}}\left(z_{n}\right) \rightarrow V_{0}$, and since $V(\xi) \geq u(\xi)$ we conclude from the lemma that for some $\rho_{0}>0, \Delta\left(\xi, \rho_{0}\right) \subseteq \Delta\left(\zeta_{n}, \rho\left(\zeta_{n}\right)\right)$ for all large $n$. Being uniformly bounded there, $v_{\varsigma_{n}}(z)$ has a subsequence which converges uniformly in $\Delta\left(\xi, \frac{1}{2} \rho_{0}\right)$, and therefore (for the subsequence) $v_{\zeta_{n}}(\xi) \rightarrow V_{0}$. Hence $V(\xi) \geq V_{0}$, a contradiction.

To see that $V(z)$ is subharmonic, fix $\xi$ and distinguish two cases, the first of which is $V(\xi)=u(\xi)$. For all positive $r<\rho(\xi)$

$$
V(\xi)=u(\xi)=v_{\xi}(\xi)=\frac{1}{2 \pi} \int_{-\pi}^{\pi} v_{\xi}\left(\xi+r e^{i \theta}\right) d \theta \leq \frac{1}{2 \pi} \int_{-\pi}^{\pi} V\left(\xi+r e^{i \theta}\right) d \theta .
$$

In the second case $V(\xi)>u(\xi)$, and then there is a sequence $\zeta_{n}$ such that $v_{\zeta_{n}}(\xi) \rightarrow$ $V(\xi)$. From the lemma it follows that there must be a neighborhood of $\xi$ contained in $\Delta\left(\zeta_{n}, \rho\left(\zeta_{n}\right)\right)$ for all large $n$, within which a subsequence of $v_{\zeta_{n}}(z)$ converges uniformly to some harmonic limit $g(z) \leq V(z)$. Hence for all small positive $r$

$$
V(\xi)=g(\xi)=\frac{1}{2 \pi} \int_{-\pi}^{\pi} g\left(\xi+r e^{i \theta}\right) d \theta \leq \frac{1}{2 \pi} \int_{-\pi}^{\pi} V\left(\xi+r e^{i \theta}\right) d \theta .
$$

PROOF OF (2.3). Let $w=e^{i \theta}$ be a point where $u(z)$ has a limit $u(w)$. We show that $\varlimsup V(z) \leq u(w)$; the other inequality $\lim V(z) \geq u(w)$ is proved similarly.

Given $\varepsilon>0$ let $\delta$ be such that $|u(z)-u(w)|<\varepsilon$ for all $z$ in $G=\Delta(0,1) \cap \Delta(w, \delta)$. Since $h_{2}(z)-h_{1}(z)$ has finite nontangential limits a.e., and $u(z)$ is between $h_{1}(z)$ and $h_{2}(z)$, it may be supposed that $\delta$ is chosen so that $h_{2}(z)$ is bounded (by $N$ say) on $\Delta(0,1) \cap \partial G$.

Let $D$ be the half-disc with center $w$ and radius $\delta$ which contains $G$, and for $z$ in $D$ define

$$
J(z)=\frac{2}{\pi}(N+\varepsilon)(\pi-\theta)+\frac{2}{\pi}(u(w)+\varepsilon)\left(\theta-\frac{1}{2} \pi\right),
$$

where $\theta=\theta(z)$ is the angle subtended at $z$ by the diameter of $D . J(z)$ is harmonic in $D$ with boundary values $u(w)+\varepsilon$ on the diameter and $N+\varepsilon$ elsewhere. Now if $z$ is in $D$ and $|z-\varsigma|<\rho(\varsigma)$, then $v_{\varsigma}(z) \leq J(z)$. The boundary of $D \cap \Delta(\zeta, \rho(\varsigma))$ consists of at most two circular arcs, one of which is contained in $D$, the other being part of the semicircumference of $D$, and on the first of these $v_{\zeta}(z)=u(z)<u(w)+\varepsilon \leq J(z)$, while on the other $v_{\varsigma}(z) \leq h_{2}(z) \leq N<J(z)$. Hence $V(z) \leq J(z)$ in $D$ which gives $\varlimsup \lim V(z) \leq u(w)+\varepsilon$ for any $\varepsilon>0$.

3. Proof of Theorem 1. $V(z)-W(z)$ is a nonnegative subharmonic function bounded above by $h_{2}(z)-h_{1}(z)$ and so can be represented as

$$
V(z)-W(z)=H(z)+K(z),
$$

where $H(z)$ is the least harmonic majorant of $V(z)-W(z)$ and $K(z)$ is a nonpositive subharmonic function with radial limits 0 a.e. [6, p. 172]. It follows from (2.3) that $H(z)$ has radial limits 0 a.e., and is, from (ii) of Theorem 1, bounded along every radius. From a uniqueness theorem due to Lohwater $[3], H(z) \equiv 0$. Since the 
left-hand side of (3.1) is nonnegative while the right-hand side is nonpositive both are zero, i.e. $V(z)=W(z)=u(z) . u(z)$ is thus harmonic, being subharmonic and superharmonic.

4. Proof of Theorem 2. The first part of the proof borrows from Lohwater's argument [3]. Let $\mu(t)$ be the nondecreasing function occurring in the Herglotz representation of $h$, and let $\mu(t)=\nu(t)+\sigma(t)$ be its Lebesgue decomposition, where $\nu(t)$ is absolutely continuous and $\sigma(t)$ is singular. Lohwater has shown [4] that if $\mu(t)$ is discontinuous at $T$ then $h\left(r e^{i T}\right) \rightarrow+\infty$ as $r \rightarrow 1$. It follows that if (1.2) holds for every $\theta$ then $\sigma(t)$ is continuous and so, according to an observation by Saks [5, p. 128], either $\sigma^{\prime}(t)=+\infty$ on an uncountable set or else $\sigma(t)$ is constant. Under the first alternative $h(z)$ has radial limits $+\infty$ on an uncountable set, which is contrary to (1.2). Thus $\sigma(t)$ is constant, which gives (1.3) with $H(t)=\nu^{\prime}(t)$.

Assume now that (1.4) fails for some $\theta$, which may be taken to be 0 . There is then a sequence $\delta_{n} \rightarrow 0$ such that

$$
\int_{-\delta_{n}}^{\delta_{n}} H(t) d t \geq 2 n \delta_{n}
$$

and therefore, for $\delta_{n} \leq t \leq 2 \delta_{n}$,

$$
\int_{-t}^{t} H(t) d t \geq n t
$$

Integration by parts gives

$$
h(r)=\frac{1}{2 \pi} \int_{-\pi}^{\pi} H(t) d t \cdot \frac{1-r}{1+r}+\frac{1}{2 \pi} \int_{-\pi}^{\pi}\left\{\int_{0}^{t} H(s) d s\right\} \frac{2 r\left(1-r^{2}\right) \sin t}{\left(1-2 r \cos t+r^{2}\right)^{2}} d t
$$

and the second term on the right is, for all large $n$,

$$
\begin{aligned}
\frac{1}{2 \pi} \int_{0}^{\pi} & \left\{\int_{-t}^{t} H(s) d s\right\} \frac{2 r\left(1-r^{2}\right) \sin t}{\left(1-2 r \cos t+r^{2}\right)^{2}} d t \\
& \geq \frac{1}{\pi} r\left(1-r^{2}\right) n \int_{\delta_{n}}^{2 \delta_{n}} \frac{t \sin t}{\left\{(1-r)^{2}+4 r \sin ^{2} \frac{1}{2} t\right\}^{2}} d t \\
& \geq \frac{1}{\pi} r\left(1-r^{2}\right) n \delta_{n}^{2} \sin \delta_{n}\left\{(1-r)^{2}+4 r \sin ^{2} \delta_{n}\right\}^{-2} \\
& \geq 2 \pi^{-2} r\left(1-r^{2}\right) n \delta_{n}^{3}\left\{(1-r)^{2}+4 r \delta_{n}^{2}\right\}^{-2}
\end{aligned}
$$

Let $r_{n}$ be the solution of $\left(1-r_{n}\right) r_{n}^{-1 / 2}=2 \delta_{n}$ which approaches $1-$ as $n \rightarrow \infty$. Then, from (4.1), $h\left(r_{n}\right) \geq \frac{1}{16} \pi^{-2} n r_{n}^{-1 / 2}\left(1+r_{n}\right)+o(1) \rightarrow+\infty$ as $n \rightarrow \infty$, contradicting (1.2).

Conversely, suppose that $h(z)$ is given by (1.3) and that (1.4) holds for every $\theta$. Since $t^{-1} \int_{0}^{t} H(s) d s$ is bounded for all $t \in(-\pi, \pi)$, we obtain from (4.1)

$$
h(r) \leq o(1)+O\left\{\left(1-r^{2}\right) \int_{-\pi}^{\pi} \frac{2 r t \sin t}{\left(1-2 r \cos t+r^{2}\right)^{2}} d t\right\}=O(1)
$$

as $r \rightarrow 1-$. By a rotation the result for any $\theta$ follows. 
5. $h^{1}$ functions. It remains to justify the remark following Theorem 2 .

For $n \geq 2$, let

$$
H_{n}(t)= \begin{cases}n^{3}, & n^{-1}-n^{-3}<t<n^{-1} \\ -n^{3}, & n^{-1}<t<n^{-1}+n^{-3} \\ 0, & \text { elsewhere }\end{cases}
$$

and let $h_{n}(z)$ be given by (1.3) with $H$ replaced by $H_{n}$. After slight simplification we obtain

$$
\begin{aligned}
0 \leq & h_{n}(r)=\frac{2 r}{\pi}\left(1-r^{2}\right) n^{3} \sin n^{-1} \\
& \times \int_{0}^{n^{-3}} \frac{\sin t}{\left[1-2 r \cos \left(n^{-1}+t\right)+r^{2}\right]\left[1-2 r \cos \left(n^{-1}-t\right)+r^{2}\right]} d t \\
\leq & \left(1-r^{2}\right) n^{-4}\left[1-2 r \cos \left(n^{-1}-n^{-3}\right)+r^{2}\right]^{-2} \\
\leq & \left(1-r^{2}\right) n^{-4}\left[\sin \left(n^{-1}-n^{-3}\right)\right]^{-4} \leq K\left(1-r^{2}\right)
\end{aligned}
$$

for some constant $K$. Also $\int_{-T}^{T} H_{n}(t) d t=n T$ when $T=n_{1}$ and $=0$ for $n^{-1}+$ $n^{-3} \leq T \leq \pi$ and $0 \leq T \leq n^{-1}-n^{-3}$.

Now define

$$
h(z)=\sum_{j=1}^{\infty} 2^{-j} h_{n_{j}}(z), \quad H(t)=\sum_{j=1}^{\infty} 2^{-j} H_{n_{j}}(z),
$$

where $n_{j}=j 2^{j}$.

Certainly $h(z) \in h^{1}$. Also, since the intervals in (5.1) for various $n$ do not overlap, we have (1.2) for $\theta \neq 0$ and, in view of (5.2), for $\theta=0$ also. Finally with $T=n_{j}^{-1}$

$$
\int_{-T}^{T} H(t) d t=\int_{-T}^{T} 2^{-j} H_{n_{j}}(t) d t=2^{-j} n_{j} T=j T
$$

so (1.4) fails at $\theta=0$.

\section{REFERENCES}

1. P. C. Fenton, Functions having the restricted mean value property, J. London Math. Soc. (2) 14 (1976), 451-458.

2. J. E. Littlewood, Some problems in real and complex analysis, Heath, Boston, Mass., 1968.

3. A. J. Lohwater, A uniqueness theorem for a class of harmonic functions, Proc. Amer. Math. Soc. 3 (1952), 278-279.

4. __ The boundary values of a class of meromorphic functions, Duke Math. J. 19 (1952), 243-252.

5. S. Saks, Theory of the integral, PWN, Warsaw, 1937.

6. M. Tsuji, Potential theory in modern function theory, Chelsea, New York.

Department of Mathematics, University of Otago, POB 56, Dunedin, New ZEALAND 